\title{
Construction of a Novel Female Sterility System for Hybrid Rice
}

\author{
Wei $\mathrm{Li}^{1+}$, Xiaoqiong Guo ${ }^{1+}$, Wenbin Wu' ${ }^{1}$, Weilin $\mathrm{Yu}^{1}$, Shichuan $\mathrm{Li}^{1}$, Di Luo ${ }^{1}$, Tianjie Wang ${ }^{1}$, \\ Qian Zhu ${ }^{1,2,3}$, Lijuan Chen ${ }^{1,2,3 *}$ and Dongsun Lee ${ }^{1,2,3 *}$ \\ ${ }^{1}$ Rice Research Institute, Yunnan Agriculture University, Kunming, China, ${ }^{2}$ State Key Laboratory for Conservation \\ and Utilization of Bio-Resources in Yunnan, Yunnan Agricultural University, Kunming, China, ${ }^{3}$ The Key Laboratory for Crop \\ Production and Smart Agriculture of Yunnan Province, Yunnan Agricultural University, Kunming, China
}

The main constraints of current hybrid rice technology using male sterility (MS) are the low yield and high labor costs of hybrid rice seed (HRS) production. Therefore, there is an urgent need for innovative new hybrid rice technology. Fortunately, we discovered a unique spontaneous sporophytic female-sterile rice mutant controlled by a single recessive locus in the nucleus. Because female-sterile mutant lines cannot produce any selfed-seeds but their pollen has totally normal functions, female sterility (FS) lines may be considered ideal pollen donors to replace the female-fertile pollen donor parent lines currently used in the HRS production. In this study, a genetically engineered FS-based system was constructed to propagate a pure transgene-free FS line using a bentazon herbicide screening. Additionally, the ability of the FS + MS (FM)-line system, with mixed plantings of FS and MS lines, to produce HRS was tested. The pilot field experiment results showed that HRS of the FM-line system was more efficient compared with the conventional FS to MS strip planting control mode. Thus, this study provides new insights into genetic engineering technology and a promising strategy for the utilization of FS in hybrid rice.

Keywords: female sterility, fst line, sexual propagation, transgene-free, FM-line system, hybrid rice

\section{INTRODUCTION}

Rice (Oryza sativa L.) is an important staple food for more than one-half of the world's population (Rao et al., 2018). However, rice cultivation still faces many challenges, such as a decrease in the farming labor force and shortages of land and water resources (Van Nguyen and Ferrero, 2006; Cheng et al., 2007). Generating hybrid rice lines, which have increased rice yields and improved tolerance levels to abiotic stresses compared with inbred rice lines, is one of the most successful applications of heterosis in the practice of agriculture and an efficient way to ensure food security (Kempe and Gils, 2011; Kim and Zhang, 2018; Wang and Deng, 2018).

To utilize hybrid vigor, two commercially viable hybrid systems utilizing cytoplasmic-genetic male sterility (CMS) and photoperiod- and thermo-sensitive genic MS genes, named as the 3-line and 2-line hybrid breeding systems, respectively, which produce first- and second generations of hybrid rice, respectively, have been developed and commercialized successfully in China (Yuan, 1966; Shi, 1985; Deng et al., 1999). In addition, a third-generation hybrid rice system has succeeded in propagating and utilizing recessive nuclear MS lines using a transgenic construct-driven nongenetically modified (GM) system called seed production technology (Chang et al., 2016; Wu et al., 2016; Basnet et al., 2019). Such a genetically engineered MS system has the ability to propagate nontransgenic MS seeds for hybrid rice seed (HRS) production and to overcome the intrinsic problems 
of the first two generations of hybrid rice systems (Wu et al., 2016; Fox et al., 2017; Zhang et al., 2018; An et al., 2019, 2020; Wan et al., 2019; Zhu et al., 2019; Song et al., 2020).

However, the benefits of the current first to third generations of hybrid rice systems are countered by increased seed production costs that result from complex HRS production procedures in the conventional strip planting mode (Zhang et al., 2017). For instance, the MS line, as the female parent, and the pollen donor line, as the male parent, should be transplanted and harvested separately. In addition to being a time-consuming, labor-intensive, and complicated process, it is also impossible to ensure that male plants have been completely removed and cannot contaminate the real hybrids (Kim et al., 2007). Therefore, using a female sterility (FS) line as the male parent has been proposed as the ideal seed production tool in combination with the current hybrid rice systems, we designed a novel HRS production system, with an FS + MS (FM)-line system, making it unnecessary to eliminate them after pollination during HRS production, in which the FS line acts as the pollen donor to replace the female-fertile pollen parent lines that cannot produce any self-pollinating or out-crossing seeds. This facilitates the high-quality and efficient mechanization of HRS production (Lee et al., 2002, 2013a; Chen et al., 2012).

Until now, only a few FS mutant genes, such as the $f_{s t}$ (Lee et al., 2002, 2013a), ptb1 (Li S.C. et al., 2013; Xia et al., 2019), and fsv1 (Liu et al., 2019; Mao et al., 2021), have been identified in rice. However, for the commercial production of hybrids, FS acting as the pollen donor parent is only feasible if the FS line is completely sterile. Fortunately, we identified a $\mathrm{B}_{\text {sister-group } \text { MADS-box }}$ gene, FEMALE-STERILE (FST) (GenBank: DQ004266.1), and the spontaneous $f_{s t}$ mutant plants are completely sterile with normal anther development and viable pollen (Lee et al., 2002; Lee et al., 2013a).

How to multiply pure FS lines derived from the $f_{s t}$ mutant efficiently and economically on a large scale needed to be determined. Several strategies for multiplying $f_{s t}$ plants have been reported previously. One involved an asexual propagation technique, such as rice dormant bud regeneration ( $\mathrm{Lv}$ et al., 2013) and rapid tiller propagation (Li S.C. et al., 2021). Another strategy is the development of a third-generation hybrid rice system (Xia et al., 2019). Furthermore, the most straightforward way to screen for sterile plants is to use a herbicide (Perez-Prat and Van Lookeren Campagne, 2002), as in the Bayer's Seedlink (Kempe and Gils, 2011) and the new two-line (Kim et al., 2007) systems, but public concern regarding GM systems restricts the utilization of this approach. Consequently, there is a strong incentive to develop a practical and fully mechanized operation that generates non-GM HRS.

As previously reported, RNA interference (RNAi) generated knock-downs of CYP81A6 gene may render rice plants susceptible to bentazon $\left(\mathrm{BTZ}^{S}\right)$, and this $\mathrm{BTZ}^{S}$ trait can be used as a marker trait to select lethal transgenic seedlings (Pan et al., 2006; Wang et al., 2012; Lu et al., 2017; He and Zhao, 2020). In this study, we chose to fundamentally redesign the target region in the conserved domain of CYP81A6 to construct a new $\mathrm{BTZ}^{S}$ lethality gene-RNAi expression cassette (BTZ-RNAi). A genetically engineered FS system for pure $f$ st lines propagation was constructed by transforming an $f_{s t}$ plant with the transgene FST $\left(F S T^{T}\right)$ for the restoration of female fertility linked with the BTZ-RNAi cassette to create lethal transgenic female-fertile plants that could be killed by spraying the herbicide BTZ. We hypothesized that if the BTZ-RNAi cassette was closely linked to $F S T^{T}$, then the activity and presence of the T-DNA in the transgenic plants would confer the $\mathrm{BTZ}^{S}$ phenotype and restore female fertility. Subsequently, we also tested a novel FM-line system for HRS production, with mixed plantings of FS and MS lines in different ratios and modes, which would facilitate the high-quality and efficient mechanization of HRS production.

\section{MATERIALS AND METHODS}

\section{Plant Materials and Growth Conditions}

The japonica rice (O. sativa ssp. japonica) variety Ilmibyeo and the homozygous fst line Yunling319FS, containing the $R f-1$ gene, were used in this study. All the plants were planted in a greenhouse of the Rice Research Institute, Yunnan Agricultural University, Kunming $\left(25.1^{\circ} \mathrm{N}, 102.7^{\circ} \mathrm{N}, 1,950\right.$ masl). In summer, the plants were grown in the greenhouse under natural conditions. In winter, the plants were grown in the greenhouse maintained at average day and night temperatures of over 30 and $20^{\circ} \mathrm{C}$, respectively, with 12 -h light/12-h dark cycles.

\section{Construction of Plant Expression Vectors}

To construct the expression vector AT72 ( $p 35 S: B T Z-R N A i)$, a $304 \mathrm{bp}$ fragment of the conserved domain of the $\mathrm{BTZ}^{S}$ lethality gene CYP81A6 (Os.11193) was amplified by polymerase chain reactions (PCR) from "Nipponbare" seedling stage leaf blade cDNA using the primer pair BTZRi-F/R (Supplementary Table 1). The fragment was then inserted into the dTA3 vector to form a hairpin structure, and an intron fragment containing $85 \mathrm{bp}$ was used as the linker, which was released from the dTA3 vector by digestion with SalI. The hairpin structure was introduced into a SalI-predigested HPE203 binary vector between the $35 \mathrm{~S}$ promoter and the nopaline synthase (NOS) terminator.

To produce the $F S T^{T}$ gene expression cassette, we first amplified a 549 bp fragment from cDNA of "Nipponbare" taken at 5 days after pollination using the primer pair FT1-F/R (Supplementary Table 1) and a 647 bp fragment from "Nipponbare" genomic DNA using primer pair FT2-F/R (Supplementary Table 1). The upstream region of FST (2,289 bp) was amplified using the primer pair FTP1-F/R (Supplementary Table 1) from "Nipponbare" genomic DNA, and the NOS terminator fragment (314 bp) was amplified using the primer pair NOS1-F/R (Supplementary Table 1) from the HPE203 binary vector. These four fragments were fused together using overlapping extension PCR and inserted into the dTA3 vector. The obtained plasmid vector was digested with $B s m \mathrm{BI}$, and the $F S T^{T}$ gene expression cassette (3,704 bp) was recovered. This was then cloned into the AT72 vector, which had been predigested with EcoRI and XbaI. This resulted in the expression vector AT73 (pFST:FST//p35S:BTZ-RNAi) (Supplementary Figure 1). AT72 and AT73 constructs were confirmed by sequencing 
before being transformed into Agrobacterium tumefaciens strain EHA105. Hygromycin resistance gene $\left(H y g^{R}\right)$ is present on the backbone of the HPE203 binary vector, which allows for the subsequent selection of transgenic calli and plants using hygromycin $\mathrm{B}$.

\section{Agrobacterium-Mediated Rice Transformation}

The AT72 construct was transformed into rice calli, which were induced from mature embryos of the japonica rice line Ilmibyeo, and the construct AT73 was introduced into the young panicleor anther-derived calli of the homozygous $f$ st line Yunling319FS.

To obtain mature embryo-derived calli, mature seeds were dehusked and sterilized by incubation in 70\% ethanol for $3 \mathrm{~min}$ and then in $1.5 \%(\mathrm{w} / \mathrm{v}) \mathrm{NaClO}$ for $30 \mathrm{~min}$ with shaking. They were washed with sterile water five times, and the seeds were cultured on the N6D medium (N6 with $30 \mathrm{~g} / \mathrm{L}$ sucrose, $2 \mathrm{mg} / \mathrm{L}$ 2,4-dichlorophenoxyacetic, and $2.4 \mathrm{~g} / \mathrm{L}$ phytagel, $\mathrm{pH} 5.7$ ) at $30^{\circ} \mathrm{C}$ in darkness. After 3 weeks, proliferated calli were subcultured on fresh N6D medium for 3 days and then used for transformation.

To obtain the anther-derived calli, rice panicles at the booting stage were collected and incubated at low temperature $\left(10^{\circ} \mathrm{C}\right)$ for 10 days under dark conditions. After a cold pretreatment, the anthers were cultured on the CM6 medium (N6 with $70 \mathrm{~g} / \mathrm{L}$ sucrose, $1 \mathrm{~g} / \mathrm{L}$ yeast extract, $0.2 \mathrm{mg} / \mathrm{L} \mathrm{2,4-}$ dichlorophenoxyacetic, $1 \mathrm{mg} / \mathrm{L}$ naphthaleneacetic, and $10 \mathrm{~g} / \mathrm{L}$ agar, $\mathrm{pH}$ 5.7) under aseptic conditions and then incubated in the dark at $30^{\circ} \mathrm{C}$. At 3 weeks after inoculation, calli growth was observed from anthers, and the formed calli were sub-cultured on fresh N6D medium for another 3 days before being used for transformation.

To obtain the young panicle-derived calli, young panicles with spikelets were placed on the surface of N6D medium under aseptic conditions and incubated for 3 weeks in the dark at $30^{\circ} \mathrm{C}$. Proliferating calli were subcultured on fresh N6D medium for another 3 days and were then used for transformation.

The constructs AT72 and AT73 were transformed independently into rice calli using Agrobacterium tumefaciens. Transgenic calli and plants with $H_{y g}{ }^{R}$ were selected and subsequently transplanted.

\section{Identification of Transgenic Plants by Leaf PCR}

Hygromycin resistance gene was employed as a selectable marker, and the transformants were screened by PCR amplification using standard amplification conditions ( 35 cycles of $20 \mathrm{~s}$ at $94^{\circ} \mathrm{C}$, $20 \mathrm{~s}$ at $58^{\circ} \mathrm{C}$, and $40 \mathrm{~s}$ at $72^{\circ} \mathrm{C}$ ) with the following primer pair HpHmMAS2-F/R (Supplementary Table 1). The PCR products were examined by agarose gel electrophoresis.

\section{Phenotypic Identification and Microscopy}

Plants and seeds were photographed using a Nikon D7500 digital camera. The seed setting rate (SSR) was estimated as the filled grain number per panicle divided by the total number of grains per panicle that includes the empty grains. The traits were measured as the mean $\pm \mathrm{SD}(n=10)$, and values of $p$ were based on $t$-tests performed using $\mathrm{R}$ software (version $4.0 .0^{1}$ ).

\section{Greenhouse Evaluation of Sensitivity to Bentazon}

Tillers of $\mathrm{T}_{0}$ plants were separated and grown as two sub-plants. One normally growing sub-plant of each $\mathrm{T}_{0}$ plant was treated with a BTZ solution $(1,200 \mathrm{mg} / \mathrm{L})$ by spraying until droplets were visible on the leaves. At the three-leaf stage, the $\mathrm{T}_{1}$ seedlings were sprayed with BTZ solution $(1,200 \mathrm{mg} / \mathrm{L})$ until droplets were visible on the leaves. BTZ (48\% solution) was obtained from Jiangsu BASF Limited (Jiangsu, China). Before the herbicides were sprayed on the rice plants, the field was drained to remove any field surface water. Three replicates of each rice line were evaluated for $\mathrm{BTZ}^{S}$.

\section{Isolation of the Flanking Sequence of T-DNA}

The flanking sequence of T-DNA was isolated by inverse PCR to analyze the integration feature of the AT73 construct on the rice genome. One microgram of rice genomic DNA was digested with restriction endonuclease Acc65I, KpnI, or Cfr42I and then self-ligated with T4 DNA ligase. Subsequently, relinearization of the DNA by restriction endonuclease HandIII and PCR was carried out, in which $0.5 \mu \mathrm{l}$ of the digested product was amplified with primer pair Inver1-F/R (Supplementary Table 1). The PCR was performed following the amplification system for TaKaRa LA Taq DNA polymerase provided by the manufacturer. Then, products of PCR were separated by electrophoresis, recovered, and sequenced. The sequences were analyzed by performing a Basic Local Alignment Search Tool (BLAST) search in the NCBI database and Rice Genome Annotation Project database to investigate the integration feature of the AT73 construct on the rice genome.

\section{Hybrid Rice Seed Production Based on the FM-Line System}

To test pollen viability, hybrid vigor potential, and the commercial viability of $\mathrm{T}_{1}$ transgene-free FS plants from AT73 transformants, they were taken as the male parent and were crossed to the elite Dian-type CMS line Hexi42A by hand emasculation and hand pollination. The resultant $F_{1}$ hybrids were grown in a greenhouse.

A pilot experiment for HRS production was conducted to test the application potential of the FS+MS (FM)-line system. The transgene-free FS line 319FS was taken as the male parent and was mixed-planted with the elite Dian-type CMS line H479A, which has a similar growth duration, for HRS production at three different mixed-planting ratios and three different cultivation modes. The three cultivation modes used were as follows: the conventional strip planting mode as the control treatment, in which the seedlings were grown 30 days and then transplanted separately with one row of the transgene-free FS line and five rows of the MS line; the random mixed-planting mode, in which

${ }^{1}$ https://www.R-project.org/ 


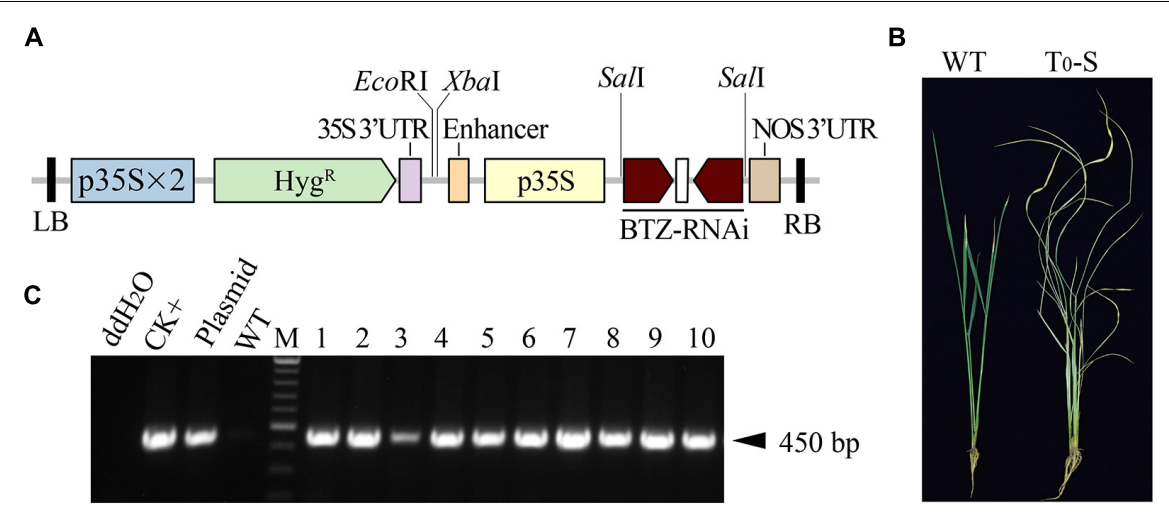

FIGURE 1 | PCR screening and BTZ ${ }^{S}$ testing of $\mathrm{T}_{0}$ transformants carrying the AT72 T-DNA fragment. (A) Schematic of the T-DNA region of the AT72 vector. p35S, cauliflower mosaic virus $35 \mathrm{~S}$ promoter; p35S $\times 2$, double $35 \mathrm{~S}$ promoter; Hyg ${ }^{R}$, hygromycin resistance gene; $35 \mathrm{~S} 3^{\prime}-\mathrm{UTR}$, the $3^{\prime}-$ UTR of the $35 \mathrm{~S}$ promoter; BTZ-RNAi, the inverted repeat sequence of the 304 bp fragment of CYP81A6; LB and RB, left and right borders of the T-DNA, respectively; NOS $3^{\prime}-$ UTR, the $3^{\prime}-U T R$ of Nos; EcoRl, Sall and Xbal are restriction enzyme sites. (B) Symptoms of a wild type (WT) Ilmibyeo sub-plant and a transgenic $T_{0} B T Z^{S}$ sub-plant ( $T_{0}-S$ ) 7 days after a BTZ foliar spray. (C) Agarose gel electrophoresis of PCR amplicons was created using $\mathrm{Hyg}^{R}$-specific primers. CK+: transgenic positive plant; Plasmid: HPE203 plasmid DNA with Hyg ${ }^{R}$; M: Thermo Scientific GeneRuler DNA Ladder Mix (SM0333); 1-10: To transgenic plants; UTR: untranslated region.

the seedlings of the FS and MS lines were grown 30 days and then mixed and transplanted randomly with one seedling per hill manually in 1:3,1:4, and 1:5 ratios; the hill mixed-planting mode, in which the seedlings were grown 30 days and then transplanted manually at four seedlings per hill, with each hill containing one plant of the transgene-free FS line and three plants of the MS line. The pilot field experiments were carried out on the experimental fields of Yuanyang, Yunnan Province $\left(23.1^{\circ} \mathrm{N}, 102.4^{\circ} \mathrm{N}, 260 \mathrm{masl}\right)$. The experimental plot area was $25 \mathrm{~m}^{2}$ for each treatment and was conducted using a random block design with three replications. At the maturity stage, the panicle SSR of the maternal plants was recorded from more than 100 panicles randomly in each plot. The results were measured as the means \pm SDs of three replicates, and values of $p$ were based on $t$-tests performed using $\mathrm{R}$ software (version 4.0.0).

\section{RESULTS}

\section{Construction of a Transgene Seedling Lethality System With Bentazon}

Because the $\mathrm{BTZ}^{S}$ trait created by the RNAi knock-down of CYP81A6 can be used to isolate transgene-free $\mathrm{T}_{1}$ rice plants, we chose to fundamentally redesign the target region of the conserved domain in CYP81A6 (Supplementary Figures 2A,B) to construct a new BTZ-RNAi vector, AT72 (p35S:BTZ-RNAi) (Figure 1A). The calli derived from mature embryos of japonica rice c.v. Ilmibyeo were co-cultivated with Agrobacterium tumefaciens harboring the AT72 vector, and $10 \mathrm{BTZ}^{S} \mathrm{~T}_{0}$ plants $\left(\mathrm{T}_{0}-\mathrm{S}\right)$ were obtained from different calli (Figure 1B). Furthermore, these 10 plants were assessed by PCR using specific $H y g^{R}$ primers, and a PCR band of the expected 450 bp was observed in all the $\mathrm{T}_{0}$ plant samples (Figure 1C).

To evaluate the availability of the BTZ-RNAi expression cassette, a total of 500 plants of the $\mathrm{T}_{1}$ progeny derived from five
$\mathrm{T}_{0}-\mathrm{S}$ lines were further tested (\#1, \#2, \#3, \#4, and \#5). The fourleaf seedlings of the wild-type (WT) control (Figure 2A) and $\mathrm{T}_{1}$ progeny (Figure 2B and Supplementary Figure 3A) were tested by spraying them with BTZ. After approximately 7 days, some $\mathrm{T}_{1}$ seedlings showed the $\mathrm{BTZ}^{S}$ phenotype and eventually died $\left(\mathrm{T}_{1}\right.$ $\mathrm{S})$, whereas other $\mathrm{T}_{1}$ seedlings remained resistant to $\mathrm{BTZ}\left(\mathrm{BTZ}^{R}\right)$ and were not visibly affected $\left(\mathrm{T}_{1}-\mathrm{R}\right)$. To evaluate the reliability of the transgene seedling lethality system, we used PCR with specific $H y g^{R}$ primers to screen all the $\mathrm{T}_{1}-\mathrm{R}$ plants from the $\mathrm{T}_{0}-\mathrm{S}$ lines, and, as expected, no PCR bands were observed in any of the $\mathrm{T}_{1}$-R plants (Figure 2C and Supplementary Figure 3B). Thus, we found that the $\mathrm{BTZ}^{R}$ plants were matched perfectly with the PCR results. To further investigate the heritability and stability of the BTZ-RNAi expression cassette in rice, $\mathrm{T}_{0}-\mathrm{S}$ line \#3 was selected as the male parent and cross-pollinated with "Hexi 42A," an elite CMS line. The two $\mathrm{F}_{1} H y g^{R}$ positive plants as assessed by PCR also exhibited the $\mathrm{BTZ}^{S}$ phenotype, whereas the two $\mathrm{F}_{2}$ populations (\#6 and \#7)performed PCR using $H y g^{R}$-specific primers, but no fragments were amplified in the total $188 \mathrm{~F}_{2}-\mathrm{R}$ plants (Figures 2D,E and Supplementary Figure 3). Thus, the expression vector AT72 appears to enable the establishment of an effective and accurate transgene seedling lethality system for screening transgene-free plants by spraying them with BTZ.

\section{Development of a Genetically Engineered Female Sterility System Based on the Transgenic Seedling Lethal Cassette}

Because FST is a sporophytic female fertility gene in rice, a hemizygous $F S T^{T}$ in the fst mutant plant that can fully restore female fertility. The $\mathrm{T}_{0}$ transgenic plants $\left(F S T^{T} / f_{s t}\right)$ were allowed to self-pollinate, and the resulting $\mathrm{T}_{1}$ progeny should show the segregation of transgenic female-fertile and transgene-free FS plants. To obtain all transgene-free FS seedlings from $\mathrm{T}_{1}$ progeny efficiently and economically, the linkage expression vector AT73 ( $p$ FST:FST//p35S:BTZ-RNAi) was constructed, containing the 


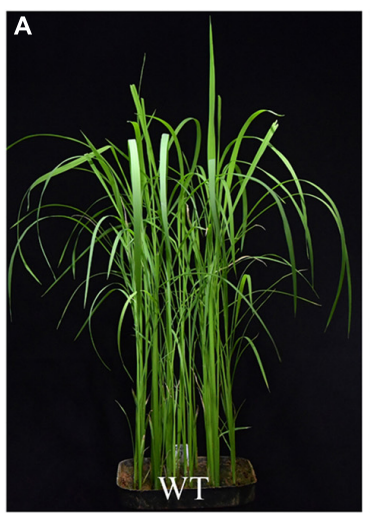

C

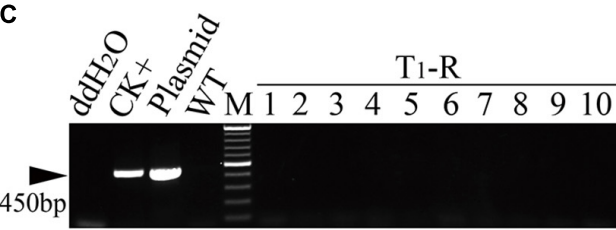

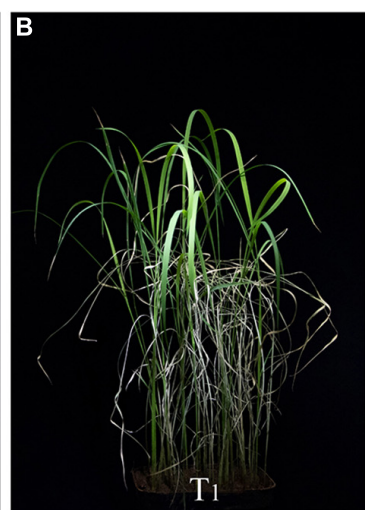

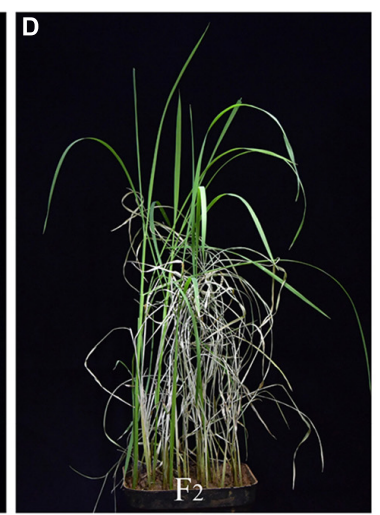

E

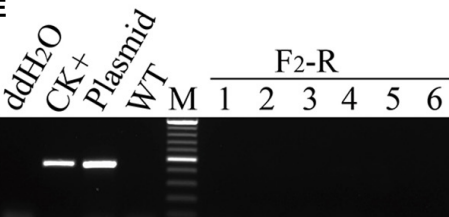

FIGURE 2 | Screening of transgene-free plants from segregation progeny of independent transgenic $T_{0}$ lines carrying the AT72 T-DNA fragment. (A,B) Symptoms of wild-type (WT) Ilmibyeo (A), transgenic $T_{1}$ progeny (\#1) (B). (C) PCR screening using $H y g^{R}$-specific primers for $B Z^{R}$ plants $\left(T_{1}-R\right)$ from transgenic $T_{1}$ progeny (\#1). (D) Symptoms of $F_{2}$ populations (\#7) seedlings 7 days after a BTZ foliar spray. (E) PCR screening using $H_{y g}{ }^{R}$-specific primers for BTZ ${ }^{R}$ plants $\left(F_{2}\right.$-R) from $F_{2}$ populations (\#7). CK+: transgenic positive plant; plasmid: HPE203 plasmid DNA with Hyg ${ }^{R}$; M: Thermo Scientific GeneRuler DNA Ladder Mix (SM0333). Hyg ${ }^{R}$, hygromycin resistance gene.

FST $T^{T}$ expression cassette, which consisted of the 1.8-kb FST promoter, the FST coding sequence with the first and last introns and the BTZ-RNAi expression cassette (Figure 3A). The AT73 construct was transformed into the young panicle-originated or anther-derived calli of the homozygous $f$ st line Yunling319FS using the Agrobacterium tumefaciens-mediated method to obtain hemizygous $F S T^{T} \mathrm{~T}_{0}$ plants.

Based on the expected 3:1 ratio of $\mathrm{BTZ}^{S}$ and $\mathrm{BTZ}^{R}$ seedlings, one $\mathrm{T}_{0}$ transgenic event, AT73-28, was chosen as a representative for further study. The $\mathrm{T}_{0}$ plants (by tiller propagation) were allowed to self-pollinate and displayed dominant female fertility restoration, with a greater than $70 \%$ SSR under greenhouse conditions (Figures 3B-D), and the self-pollinated mature seeds presented almost the same phenotype as normal seeds (Figure 3C). Furthermore, $\mathrm{BTZ}^{S}$ test results showed that the $\mathrm{T}_{0}$ plants $\left(\mathrm{T}_{0}-\mathrm{S}\right)$ died about 1 week later by spraying BTZ (Figure 3E), and a PCR analysis using $H y g^{R}$-specific primers confirmed that the $\mathrm{T}_{0}$ plant was transgenic (Figure 3F). The integration site of T-DNA borders in the $\mathrm{T}_{0}$ plant was analyzed by flanking sequence isolation. $\mathrm{T}_{0}$ plant AT73-28, which was analyzed three times by inverse PCR, gave the same location of integration, and T-DNA integration at 105,018 bp (GenBank Accession No. AL606639.3) at chromosome 4 in the rice genome (Figure 3G and Supplementary Figure 4).

In addition, all of the individual $\mathrm{T}_{1}$ plants of AT73-28 were analyzed by PCR using $H y g^{R}$-specific primers to determine whether they were transgenic plants or segregates without the transgene. The $H y g^{R}$-specific primers did not amplify any bands in some of the individual $\mathrm{T}_{1}$ plants (Figure $3 \mathrm{~F}$ ). Additionally, $\mathrm{BTZ}^{S}$ test results showed that all of the positive transgenic
$\mathrm{T}_{1}$ plants $\left(\mathrm{T}_{1}-\mathrm{S}: F S T^{T} /\right.$ fst and/or $\left.F S T^{T} / F S T^{T}\right)$ died within approximately 7 days of being sprayed with BTZ, whereas the negative $\mathrm{T}_{1}$ segregates $\left(\mathrm{T}_{1}-\mathrm{R}\right.$ : $\left.f_{s t} / f_{s t}\right)$ without the transgene survived as expected (Figure $3 \mathrm{E}$ ).

Thus, the transgenic event AT73-28 appeared to produce heterozygotes $\left(F S T^{T} / f_{s t}\right)$ of the $F S T^{T}$ loci, and the presence of the T-DNA cassettes, such as the BTZ-RNAi, FST ${ }^{T}$, and $H y g^{R}$ expression cassette ( $p F S T: F S T / / p 35 S: B T Z-R N A i)$, was tightly linked in $\mathrm{T}_{0}$ plant. Therefore, this system may efficiently propagate the transgene-free FS line $\left(f_{s t} / f_{s t}\right)$ by self-pollination of the genetically engineered FS line and transgene seedling lethality screening at the seedling stage in a greenhouse.

\section{Application of the FM-Line System to Hybrid Rice Seed Production}

Because the transgene-free $T_{1}$ plants were completely FS, they could be cross-pollinated with MS plants for the mechanized production of HRS. The transgene-free FS line 319FS of the $T_{1}$ plants $\left(T_{1}-R\right)$ from AT73-28 was used as the pollen donor to cross with a Dian-type CMS line "Hexi42A," and their hybridization produced a normal SSR as expected (Figures 4A,B). The $\mathrm{F}_{1}$ hybrids were planted with proper management, and they exhibited the expected heterosis and ideal architecture with good performance (Figure 4C). Thus, the transgene-free FS line 319FS may be used as the pollen donor parent and applied to improve HRS production.

In a pilot experiment, we tested the mean outcrossing SSR of the MS plants by establishing three different FS line 319FS and MS line H479A mixed-planting modes (Figure 4D). There was a 


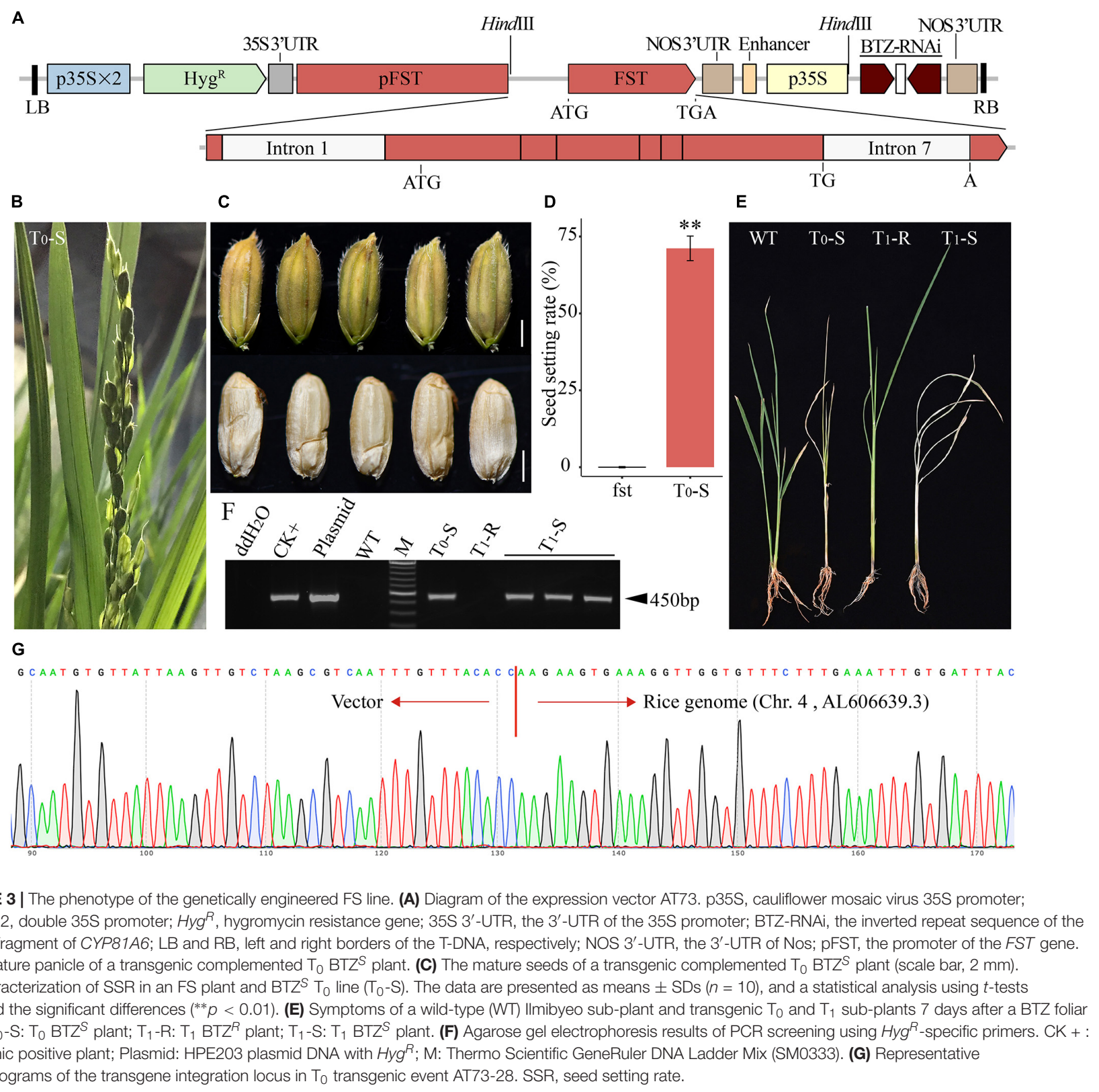

significant difference in panicle SSR of the MS plants compared with the conventional strip planting mode (Figure 4E). The mean outcrossing SSRs of MS plants from the random 1:3, 1:4, and 1:5 FS and MS lines' mixed-planting modes were 42.45, 54.07, and $51.47 \%$, respectively. In the hill 1:3 of FS and MS lines' mixed-planting modes, the mean outcrossing SSR of MS plants was $69.77 \%$, whereas that of the MS line of the conventional 1:5 FS and MS lines' strip planting control mode was 36.49\%. Thus, there is great potential for considering the FS lines as the ideal pollen donors to replace the female-fertile pollen parent lines (i.e., restorer lines) currently used in 2-, and 3-line systems of HRS production. Taken together, it was further illustrated that the genetically engineered FS system (Figure 5A) and FMline system (Figure 5B), and mixed-plantings of FS and MS lines, greatly reduced labor for transplanting and harvesting separately male parents.

\section{DISCUSSION}

In the present study, the fst mutant did not produce any selfpollinating or outcrossing seeds, whereas it showed normal vegetative growth and pollen fertility. During the implementation of the hybrid rice breeding projects at Yunnan Agricultural University, over a dozen perennial FS lines containing the CMS restorer gene (Rf-1) (Komori et al., 2004) in different genetic backgrounds were bred via complex hybridization and self-breeding based on the fst mutant (Li S.C. et al., 2021; 


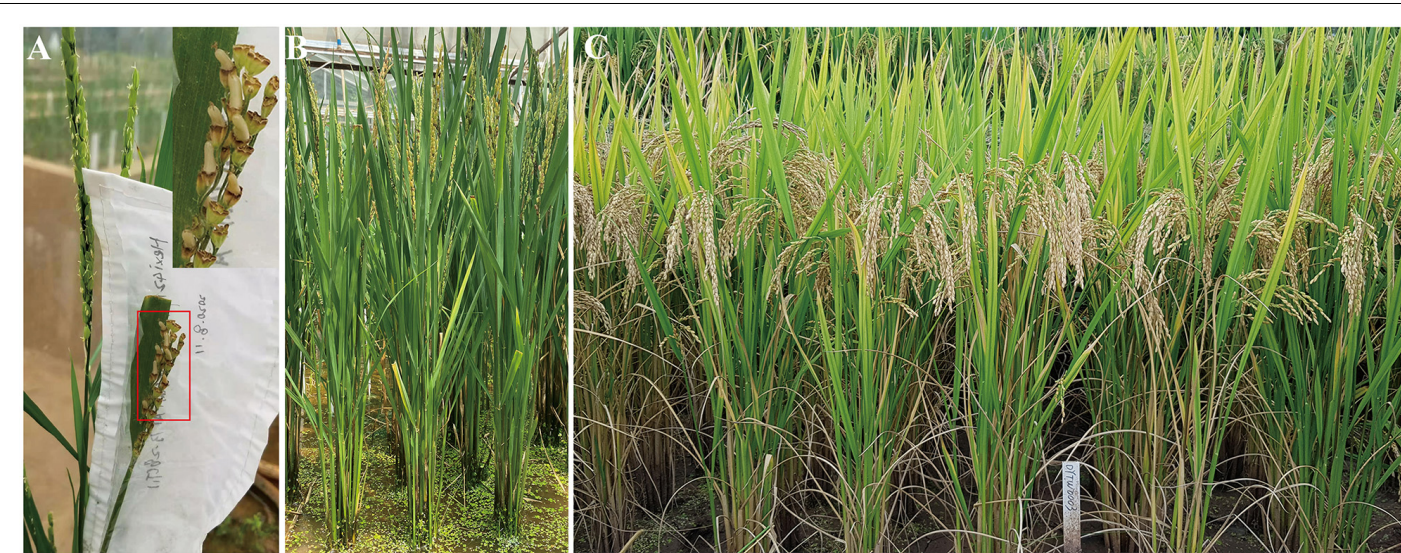

D

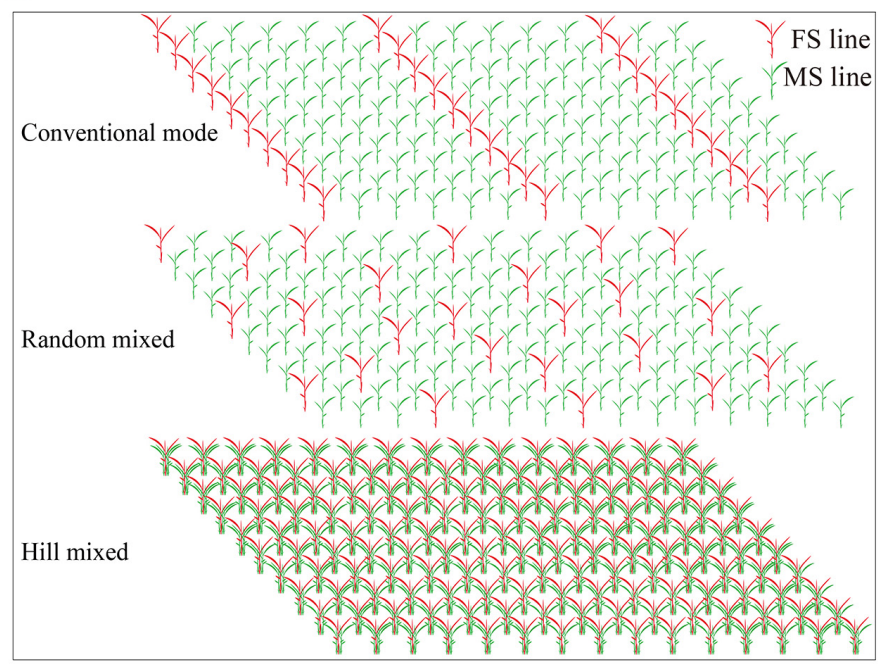

E 80

Conventional strip planting

Random mixed-planting

Hill mixed-planting

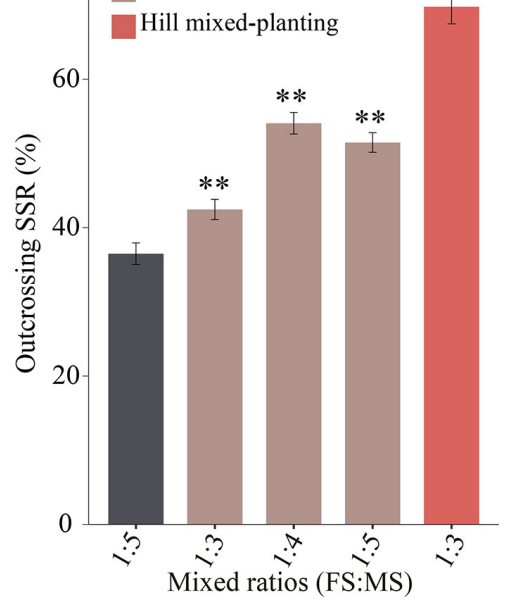

FIGURE 4 | HRS production is based on the FM-line system. (A) The seed setting of the maternal 'Hexi42A' plant is pollinated by pollen from the transgene-free FS line 319FS. (B) Morphology of transgene-free FS line 319FS. (C) Plant-type and heterosis observations of the hybrid $F_{1}$ generation of Hexi42A/319FS. (D) Diagram of the FM-line system using three different mixed-planting methods for HRS production. (E) Statistical data of the outcrossing SSR of the maternal plants in HRS production using the FM-line system. The data are presented as means \pm SDs $(n=100)$, and a statistical analysis using $t$-tests indicated the significant differences $\left({ }^{* *} p<0.01\right)$. HRS, hybrid rice seed; SSR, seed setting rate.

Yu et al., 2021; Zhu et al., 2021). Currently, the de novo development of FS lines in different restorer backgrounds using the CRISPR-Cas9 genome editing technology is underway (our unpublished data) which could $y$ assist in developing more efficient FS lines to be broadly utilized for hybrid rice breeding. At present, to propagate a large number of plants and/or seeds of pure transgene-free FS line for facilitating the mechanization of HRS production, we designed a novel biotechnology-based genetically engineered FS system (Figure 5A) and FM-line system (Figure 5B).

The FM-line system is superior to the traditional HRS production method and other biotechnology-based systems in several aspects. First, relative to the other FS mutants, such as ptb1 (Li S.C. et al., 2013) and $f s v 1$ (Liu et al., 2019; Mao et al., 2021), here, the FS lines were created by hybridization breeding and using a natural mutant $f_{s t}$ (Lee et al., 2013a), and the plants propagated were completely sterile in different genetic backgrounds and under different environmental conditions. Second, compared with the current 2- and 3-line systems of HRS technology, the FM-line system facilitated the mechanization of high-quality and efficient HRS production using a mixed-planting and mixed-harvesting approach for FS and $\mathrm{MS}$ lines. Third, $\mathrm{BTZ}^{S}$ test results confirm that FM-line system works as efficiently as other transgene sorting systems, such as fluorescence-assisted sorting method (Aliaga-Franco et al., 2019; Xia et al., 2019), weight-based seed sorting system (Wu et al., 2021), and anthocyanin-marker-assisted sorting system (He et al., 2020), but with the advantage of using the BTZ ${ }^{S}$ trait to select lethal transgenic seedlings due to its simplicity, accuracy, and sensitivity. Finally, neither the commercial HRS produced using the transgene-free FS line resulting from the genetically engineered FS system nor the resulting commodity rice grain harvested from these hybrid plants contained transgene DNA and were, therefore, transgene-free in the FM-line system (Figure 5B). Furthermore, the genetically engineered $f$ st lines $\left(F S T^{T} / f s t\right)$ propagation by dormant buds or tillers requires 

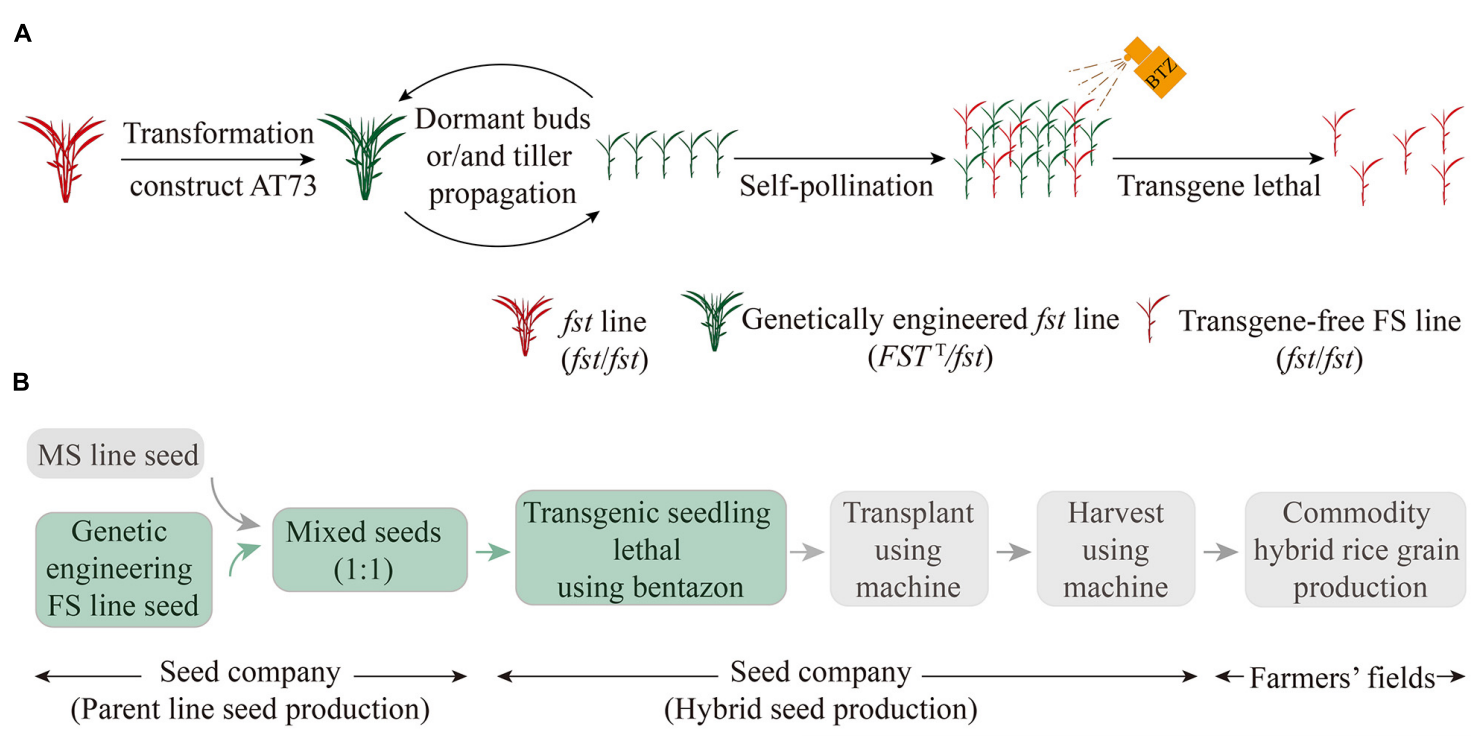

Transgenic for FM-line system Transgene-free for FM-line system

FIGURE 5 | Schematic representation of the FM-line system process for the mechanization of HRS production. (A) The genetically engineered FS system to propagate transgene-free FS lines. The red-colored plants are non-transgenic or transgene-free; the green-colored plants are transgenic. (B) The FM-line system for mechanized HRS production. HRS, hybrid rice seed.

limited acreage in greenhouse or fields together with strict spatial or temporal isolation measures would minimize the chances of out-pollination (Figure 5A).The future perspect of commercial production of HRS using FS lines focuses on the state-of-the-art developments in the seed company under the government's supervision.

In the FM-line system, other particular concerns are how to maintain and propagate a large quantity of the genetically engineered FS lines, such as the $\mathrm{T}_{0}$ line AT73-28. Prior studies have noted the importance of producing FS plants by asexual propagation based on rice dormant bud regeneration (Lee et al., 2013b) or iterative rapid tiller propagation ( $\mathrm{Li} \mathrm{W}$. et al., 2021). During the $T_{0}$ line propagation phase, rice dormant bud regeneration or rapid tiller propagation can multiply a large number of plants, and the $T_{1}$ seeds were produced by $\mathrm{T}_{0}$ plant self-pollination. During the $\mathrm{FS}$-line propagation phase, the transgenic seedlings can be selectively killed by BTZ spraying and those that remained alive represent transgenefree FS plants. Prerequisites for the FM-line system are the reliability and accuracy of transgene seeding lethality screening with BTZ. Our studies showed that all the transgenic rice plants in the $T_{0}, T_{1}$, and $F_{2}$ populations could be selectively killed by BTZ spraying, suggesting that the BTZ-RNAi expression cassette had a high screening efficiency. Additionally, previous studies have established that transgenic rice plants can be selectively killed at $100 \%$ by BTZ spraying (Lin et al., 2008; Liu et al., 2012; Lu et al., 2017). Thus, transgenic oversight is applicable only to the genetically engineered FS lines' cultivation and transgene-free FS line propagation, which requires limited acreage. Concerns regarding the evolution of herbicide resistance in natural variations can be buffered by using a rigorous screening by bentazon spraying during the different phases of HRS production. Careful analysis of the mixed FS and MS seedlings and $\mathrm{F}_{1}$ seeds for the transgene before their commercial production would minimize the potential risk of environmental contamination. In this context, we showed that the fst mutant and its derived lines can be used as transgene-free FS lines in the FMline system. The fst mutation confers stable genic-controlled FS in both indica and japonica rice. Thus, the $f$ st mutation has great potential in commercial HRS production. Our studies indicated that the FST ${ }^{T}$ expression cassette in the AT73 construct was able to restore spikelet fertility (Figure 3D). Furthermore, the rice FST is a key regulator and plays multiple functions during ovule and early seed development (Yang et al., 2012; Yin and Xue, 2012; Lee et al., 2013a). While this study clearly demonstrated that the FM-line system is feasible, more detailed studies are required to optimize the FST gene expression cassette needed to generate the genetically engineered FS lines with desirable seed development and SSR.

Our pilot experiment's results showed that different mixedplanting modes and ratios affect the yield of HRS production in the FM-line system, especially in the hill 1:3 mixed-planting mode, in which the mean outcrossing SSR of the MS line was almost double that of the conventional strip planting control mode (Figure 4E). For practical HRS production, the outcrossing SSR of MS plants should increase as a result of additional artificial supplementary pollination processes. Thus, further field experiments are required to establish the optimal proportion of FS and MS lines' mixed-planting modes in combination with additional artificial supplementary pollination processes, which would facilitate the high quality and efficient mechanization of HRS production. 


\section{DATA AVAILABILITY STATEMENT}

The original contributions presented in the study are included in the article/Supplementary Material, further inquiries can be directed to the corresponding authors.

\section{AUTHOR CONTRIBUTIONS}

DSL and LJC designed the experiments. WL and XQG performed the experiments, analyzed the data, and drafted the manuscript. QZ, WBW, WLY, SCL, DL, and TJW participated in performing the experiments. All authors reviewed and approved the manuscript for publication.

\section{FUNDING}

This study was supported by grants from the National Key Research and Development Program of China (grant

\section{REFERENCES}

Aliaga-Franco, N., Zhang, C., Presa, S., Srivastava, A. K., Granell, A., Alabadí, D., et al. (2019). Identification of transgene-free CRISPR-edited plants of rice, tomato, and arabidopsis by monitoring DsRED fluorescence in dry seeds. Front. Plant Sci. 10:1150. doi: 10.3389/fpls.2019.01150

An, X. L., Dong, Z. Y., Tian, Y. H., Xie, K., Wu, S. W., Zhu, T. T., et al. (2019). ZmMs30 encoding a novel GDSL lipase is essential for male fertility and valuable for hybrid breeding in maize. Mol. Plant 12, 343-359. doi: 10.1016/ j.molp.2019.01.011

An, X. L., Ma, B., Duan, M. J., Dong, Z. Y., Liu, R. G., Yuan, D. Y., et al. (2020). Molecular regulation of ZmMs7 required for maize male fertility and development of a dominant male-sterility system in multiple species. Proc. Natl. Acad. Sci. U.S.A. 117, 23499-23509. doi: 10.1073/pnas.201025 5117

Basnet, R., Hussain, N., and Shu, Q. Y. (2019). OsDGD2 $\beta$ is the sole digalactosyldiacylglycerol synthase gene highly expressed in anther, and its mutation confers male sterility in rice. Rice 12:66. doi: 10.1186/s12284-0190320-Z

Chang, Z. Y., Chen, Z. F., Wang, N., Xie, G., Lu, J. W., Yan, W., et al. (2016). Construction of a male sterility system for hybrid rice breeding and seed production using a nuclear male sterility gene. Proc. Natl. Acad. Sci. U.S.A. 113, $14145-14150$.

Chen, L. J., Lee, D. S., Li, C. Y., Tan, X. L., Xu, X. Z., and Zhu, Y. Y. (2012). Method of Female Sterility Gene FST for Hybrid Rice Breeding. Patent, CN101658129B. Guizhou: Yunnan Agricultural University.

Cheng, S. H., Zhuang, J. Y., Fan, Y. Y., Du, J. H., and Cao, L. Y. (2007). Progress in research and development on hybrid rice: a super-domesticate in China. Ann. Bot. 100, 959-966. doi: 10.1093/aob/mcm 121

Deng, H. F., Shu, F. B., and Yuan, D. Y. (1999). An overview of research and utilization of Annong S-1. Hybrid Rice 14, 1-3.

Fox, T., DeBruin, J., Haug Collet, K., Trimnell, M., Clapp, J., Leonard, A., et al. (2017). A single point mutation in Ms44 results in dominant male sterility and improves nitrogen use efficiency in maize. Plant Biotechnol. J. 15, 942-952.

He, Y. B., and Zhao, Y. D. (2020). Technological breakthroughs in generating transgene-free and genetically stable CRISPR-edited plants. aBIOTECH 1 , 88-96. doi: 10.1007/s42994-019-00013-x

He, Y. B., Zhu, M., Wu, J. H., Ouyang, L., Wang, R. C., Sun, H., et al. (2020). Repurposing of anthocyanin biosynthesis for plant transformation and genome editing. Front. Genome Ed. 2:607982. doi: 10.3389/fgeed.2020.60 7982

Kempe, K., and Gils, M. (2011). Pollination control technologies for hybrid breeding. Mol. Breed. 27, 417-437. doi: 10.1007/s11032-011-9555-0 nos. 2016YFD0101101-5 and 2017YFD0100205), the National Natural Science Foundation of China (grant nos. 31560115 and 31860108), the Yunnan Applied Basic Research Projects (grant no. 2015FA023), and the Yunling Super-Talent Initiative"Yunling High-End Foreign Expert" program.

\section{ACKNOWLEDGMENTS}

We thank the reviewers for the valuable advice to improve the manuscript. We are also thankful to Sadia Nadir, for helping with manuscript revision.

\section{SUPPLEMENTARY MATERIAL}

The Supplementary Material for this article can be found online at: https://www.frontiersin.org/articles/10.3389/fpls.2021. 815401/full\#supplementary-material

Kim, S. S., Jung, J. Y., Jeong, S. K., Lee, D. S., Chen, L. J., and Suh, H. S. (2007) Use of herbicide-resistant genic male sterility in hybrid rice seed production. Euphytica 156, 297-303. doi: 10.1007/s10681-006-9314-1

Kim, Y. J., and Zhang, D. (2018). Molecular control of male fertility for crop hybrid. Trends Plant Sci. 23, 53-65. doi: 10.1016/j.tplants.2017.10.001

Komori, T., Ohta, S., Murai, N., Takakura, Y., Kuraya, Y., Suzuki, S., et al. (2004). Map-based cloning of a fertility restorer gene, $\mathrm{Rf}-1$, in rice (Oryza sativa L.). Plant J. 37, 315-325. doi: 10.1046/j.1365-313X.2003.01961.x

Lee, D. S., Chen, L. J., Ha, W. G., and Suh, H. S. (2002). Single recessive genetic female sterility in rice. Int. Rice Res. Notes 27, 22-23.

Lee, D. S., Chen, L. J., Li, C. Y., Liu, Y., Tan, X. L., Lu, B. R., et al. (2013a). The Bsister MADS gene FST determines ovule patterning and development of the zygotic embryo and endosperm. PLoS One 8:e0058748. doi: 10.1371/journal. pone. 0058748

Lee, D. S., Chen, L. J., Tan, X. L., Lv, Y. G., Sun, M. J., Zhu, Y. Y., et al. (2013b). Rice Dormant Buds Regeneration Plant Breeding Methods. Patent, CN103070052B. Guizhou: Yunnan Agricultural University.

Li, S. C., Li, W. B., Huang, B., Cao, X. M., Zhou, X. Y., Ye, S. M., et al. (2013). Natural variation in PTB1 regulates rice seed setting rate by controlling pollen tube growth. Nat. Commun. 4, 1-13. doi: 10.1038/ncomms3793

Li, S. C., Liu, F. G., Wang, T. J., Wang, J. B., Luo, D., Yu, W. L., et al. (2021). Characterization of rapid tiller propagation and iterative regeneration potential in rice female sterile lines. Mol. Plant Breed. [Epub ahead of print].

Li, W., Zhu, Q., Guo, X. Q., Luo, F., Wu, W. B., Yu, W. L., et al. (2021). Marker assisted breeding of the genic female sterile restorer line 'Yunling 319FS' of rice (Oryza sativa L .). Mol. Plant Breed. [Epub ahead of print].

Lin, C. Y., Fang, J., Xu, X. L., Zhao, T., Cheng, J. A., Tu, J. M., et al. (2008). A built-in strategy for containment of transgenic plants: creation of selectivity terminable transgenic rice. PLoS One 3:e1818. doi: 10.1371/journal.pone.0001818

Liu, C. Y., Li, J. J., Gao, J. H., Shen, Z. C., Lu, B. R., and Lin, C. Y. (2012). A built-in mechanism to mitigate the spread of insect-resistance and herbicidetolerance transgenes into weedy rice populations. PLoS One 7:e0031625. doi: 10.1371/journal.pone.0031625

Liu, H. L., Wang, R. H., Mao, B. G., Zhao, B. R., and Wang, J. B. (2019). Identification of lncRNAs involved in rice ovule development and female gametophyte abortion by genome-wide screening and functional analysis. BMC Genomics 20:6. doi: 10.1186/s12864-019-5442-6

Lu, H. P., Liu, S. M., Xu, S. L., Chen, W. Y., Zhou, X., Tan, Y. Y., et al. (2017). CRISPR-S: an active interference element for a rapid and inexpensive selection of genome-edited, transgene-free rice plants. Plant Biotechnol. J. 15, 1371-1373. doi: $10.1111 /$ pbi. 12788

Lv, Y. G., Gan, S. X., Feng, D. D., Dong, C. W. H., Xiong, H. B., Zhu, Q., et al. (2013). The establishment of asexual propagation system based on rice dormant buds 
regeneration. Mol. Plant Breed. 11, 1032-1041. doi: 10.5376/mpb.cn.2013.11. 0006

Mao, B. G., Zheng, W. J., Huang, Z., Peng, Y., Shao, Y., Liu, C. T., et al. (2021). Rice MutL(, the MLH1-MLH3 heterodimer, participates in the formation of type I crossovers and regulation of embryo sac fertility. Plant Biotechnol. J. 19, 1443-1455. doi: 10.1111/pbi.13563

Pan, G., Zhang, X. Y., Liu, K. D., Zhang, J. W., Wu, X. Z., Zhu, J., et al. (2006). Map-based cloning of a novel rice cytochrome P450 gene CYP81A6 that confers resistance to two different classes of herbicides. Plant Mol. Biol. 61, 933-943. doi: 10.1007/s11103-006-0058-Z

Perez-Prat, E., and Van Lookeren Campagne, M. M. (2002). Hybrid seed production and the challenge of propagating male-sterile plants. Trends Plant Sci. 7, 199-203. doi: 10.1016/S1360-1385(02)02252-5

Rao, G. S., Deveshwar, P., Sharma, M., Kapoor, S., and Rao, K. V. (2018). Evolvement of transgenic male-sterility and fertility-restoration system in rice for production of hybrid varieties. Plant Mol. Biol. 96, 35-51. doi: 10.1007/ s11103-017-0678-5

Shi, M. S. (1985). The discovery and study of the photosensitive recessive malesterile rice (Oryza sativa L. subsp. japonica). Sci. Agric. Sin. 2, 44-48.

Song, S. F., Wang, T. K., Li, Y. X., Hu, J., Kan, R. F., Qiu, M. D., et al. (2020). A novel strategy for creating a new system of third-generation hybrid rice technology using a cytoplasmic sterility gene and a genic male-sterile gene. Plant Biotechnol. J. 19, 251-260. doi: 10.1111/pbi.13457

Van Nguyen, N., and Ferrero, A. (2006). Meeting the challenges of global rice production. Paddy Water Environ. 4, 1-9. doi: 10.1007/s10333-005-0031-5

Wan, X., Tian, Y., Dong, Z., Ma, B., An, X., Li, J., et al. (2019). Maize genic male-sterility genes and their applications in hybrid breeding: progress and perspectives. Mol. Plant 12, 321-342. doi: 10.1016/j.molp.2019.01.014

Wang, H. Y., and Deng, X. W. (2018). Development of the "third-generation" hybrid rice in China. Genomics Proteomics Bioinform. 16, 393-396. doi: 10. 1016/j.gpb.2018.12.001

Wang, Q. Z., Fu, H. W., Huang, J. Z., Zhao, H. J., Li, Y. F., Zhang, B., et al. (2012). Generation and characterization of bentazon susceptible mutants of commercial male sterile lines and evaluation of their utility in hybrid rice production. F. Crop. Res. 137, 12-18. doi: 10.1016/j.fcr.2012.09.001

Wu, J. X., Qiu, S. J., Wang, M. L., Xu, C. J., Deng, X. W., and Tang, X. Y. (2021). Construction of a weight-based seed sorting system for the third-generation hybrid rice. Rice 14:66.

Wu, Y. Z., Fox, T. W., Trimnell, M. R., Wang, L. J., Xu, R. J., Cigan, A. M., et al. (2016). Development of a novel recessive genetic male sterility system for hybrid seed production in maize and other cross-pollinating crops. Plant Biotechnol. J. 14, 1046-1054. doi: 10.1111/pbi.12477

Xia, Y. M., Tang, N., Hu, Y. Y., Li, D., Li, S. C., Bu, X. L., et al. (2019). A method for mechanized hybrid rice seed production using female sterile rice. Rice 12:39. doi: 10.1186/s12284-019-0296-8
Yang, X. L., Wu, F., Lin, X. L., Du, X. Q., Chong, K., Gramzow, L., et al. (2012). Live and let die-the Bsister MADS-box gene OsMADS29 controls the degeneration of cells in maternal tissues during seed development of rice (Oryza sativa). PLoS One 7:e51435. doi: 10.1371/journal.pone.0051435

Yin, L. L., and Xue, H. W. (2012). The MADS29 transcription factor regulates the degradation of the nucellus and the nucellar projection during rice seed development. Plant Cell 24, 1049-1065.

Yu, W. L., Wu, W. B., Zhu, Q., Li, J., Li, W., Guo, X. Q., et al. (2021). Detection of SNP fingerprint and variation in loci related to stress resistances of genic female sterile lines in rice. Mol. Plant Breed. [Epub ahead of print].

Yuan, L. P. (1966). Male sterility in rice. Chinese Sci. Bull. 17, 185-188.

Zhang, D. F., Wu, S. W., An, X. L., Xie, K., Dong, Z. Y., Zhou, Y., et al. (2018). Construction of a multicontrol sterility system for a maize male-sterile line and hybrid seed production based on the ZmMs7 gene encoding a PHDfinger transcription factor. Plant Biotechnol. J. 16, 459-471. doi: 10.1111/pbi.1 2786

Zhang, X. H., Zuo, B., Song, Z. J., Wang, W., He, Y. C., Liu, Y. H., et al. (2017). Breeding and study of two new photoperiod- and thermo-sensitive genic male sterile lines of polyploid rice (Oryza sativa L.). Sci. Rep. 7, 1-12. doi: 10.1038/ s41598-017-15241-8

Zhu, Q., Li, W., Guo, X. Q., Luo, F., Li, S. C., Chen, L. J., et al. (2021). Breeding Method of Perennial Genic Female Sterile Lines of Rice (Oryza sativa L.). Patent, CN112715349B. Guizhou: Yunnan Agricultural University.

Zhu, T. T., Wu, S. W., Zhang, D. F., Li, Z. W., Xie, K., An, X. L., et al. (2019). Genome-wide analysis of maize GPAT gene family and cytological characterization and breeding application of ZmMs33/ZmGPAT6 gene. Theor. Appl. Genet. 132, 2137-2154. doi: 10.1007/s00122-019-03343-y

Conflict of Interest: The authors declare that the research was conducted in the absence of any commercial or financial relationships that could be construed as a potential conflict of interest.

Publisher's Note: All claims expressed in this article are solely those of the authors and do not necessarily represent those of their affiliated organizations, or those of the publisher, the editors and the reviewers. Any product that may be evaluated in this article, or claim that may be made by its manufacturer, is not guaranteed or endorsed by the publisher.

Copyright (c) $2022 \mathrm{Li}, \mathrm{Guo}, \mathrm{Wu}, \mathrm{Yu}, \mathrm{Li}$, Luo, Wang, Zhu, Chen and Lee. This is an open-access article distributed under the terms of the Creative Commons Attribution License (CC BY). The use, distribution or reproduction in other forums is permitted, provided the original author(s) and the copyright owner(s) are credited and that the original publication in this journal is cited, in accordance with accepted academic practice. No use, distribution or reproduction is permitted which does not comply with these terms. 